\title{
A Maximum Entropy Multisource Information Fusion Method to Evaluate the MTBF of Low-Voltage Switchgear
}

\author{
Jing-Qin Wang, ${ }^{1}$ Zhi-Gang Zhang, ${ }^{1}$ Ching-Hsin Wang $\mathbb{D}^{2}{ }^{2}$ and Li Wang ${ }^{1}$ \\ ${ }^{1}$ School of Electrical Engineering, Hebei University of Technology, Tianjin 300130, China \\ ${ }^{2}$ Institute of Project Management, Department of Leisure Industry Management, National Chin-Yi University of Technology, \\ Taichung 41170, Taiwan
}

Correspondence should be addressed to Ching-Hsin Wang; thomas_6701@yahoo.com.tw

Received 31 December 2017; Revised 22 March 2018; Accepted 28 March 2018; Published 7 May 2018

Academic Editor: Chris Goodrich

Copyright (c) 2018 Jing-Qin Wang et al. This is an open access article distributed under the Creative Commons Attribution License, which permits unrestricted use, distribution, and reproduction in any medium, provided the original work is properly cited.

\begin{abstract}
When analyzing the reliability of low-voltage switchgear by Bayesian method, the maximum entropy multisource information fusion method was proposed to obtain the prior information of low-voltage switchgear and then evaluate the reliability. The historical data of low-voltage switchgear was collected and organized from a manufacturer. According to the expert experience and the data, the creditability analysis and the compatibility test were presented by the Smirnov test method. Based on the high creditability and compatibility, the result of the maximum entropy multisource information fusion method is the determination of prior information. Therefore, the distribution type of the prior information was confirmed by using the maximum entropy method, and the parameter of the prior information was received by bootstrap method with MATLAB. Then the posterior distribution was obtained to evaluate the MTBF of low-voltage switchgear. Finally, the historical data of years from 2007 to 2010 was taken as prior information to illustrate the maximum entropy multisource information fusion method and to get the MTBF of lowvoltage switchgear. The evaluation result reduces the experimental period and test cost, which is an improvement for the reliability evaluation and management of low-voltage switchgear and also an improvement for other systems with simple sample data. Compared with traditional Bayesian networks, the proposed method can fuse experts experience and historical data and has advantages for the use of prior information effectively.
\end{abstract}

\section{Introduction}

About $80 \%$ of electric energy in our country is provided by low-voltage switchgear; therefore, the reliability of lowvoltage switchgear is significant for the safety and efficient operation of electric power system $[1,2]$. When analyzing the reliability by Bayesian theory, although it is a solid theory foundation for the evaluation of low-voltage switchgear that rational utilization of experience and prior information, the determination of prior information is an important problem. It has received attention at home and abroad.

Bayesian network is a commonly used tool in probabilistic reasoning of uncertainty in industrial processes [3]. Cai et al. [4-6] proposed a multisource information fusion based fault diagnosis methodology using Bayesian network, which can increase the fault diagnostic accuracy for single fault. Huang et al. [7] developed a practical approach for fatigue lifetime assessment of aircraft engine discs by combining a multisource information fusion method with a Bayesian inference technique and the method quantifies the subjective information, checks different experts' information, and fuses multiple prior distributions. Rigas et al. [8] presented a research for the use of multisource information fusion, which can currently evaluate specifically for the eye movement biometrics. Wang et al. [9] designed a framework for multisource heterogeneous information fusion in the IoT and used an experimental simulation platform to build an environmental monitoring system to assess the framework. Zhao et al. [10] investigated the empirical entropy method for right censored data, which gives better coverage probability than that of the empirical likelihood method for contaminated and censored lifetime data. Singh et al. [11] used a theoretical method based on maximum Shannon entropy framework to study the finite buffer system, and the advantage of the method is that it has 
enabled one to derive the analytical closed form generalized expression of the probability distribution of queue size in finite buffer system.

The collection of prior information is very important, when analying the reliability of low-voltage switchgear by Bayesian method. The maximum entropy principle has advantages for the use of prior information effectively [12, 13]. Multisource information fusion can fuse experts experience and historical data effectively $[14,15]$. It considers prior information and the optimal prior distribution is selected by the maximum entropy under the boundary conditions $[16,17]$. Caticha Ariel used maximum entropy to translate the information contained in the form of the likelihood into a prior distribution for Bayesian inference [18]. Kim demonstrated the method that obtains prior information by entropy [19], which showed the effectiveness of the method. Therefore, in this paper, the maximum entropy multisource information fusion method was proposed to obtain the prior information of low-voltage switchgear and then evaluate the reliability index MTBF. The maximum entropy multisource information fusion method not only considers the credibility of prior information, but also obtains reliable results from prior information completely. Firstly, the credibility of prior information was analyzed by expert experience and historical data. Secondly, the consistency test was completed by Smirnov test to check whether prior information and field test information obey the same distribution. Thirdly, the prior distribution type was known, and hyperparameters of prior distribution function were calculated by bootstrap method. At last, the a posteriori distribution was obtained, and the evaluation of MTBF was completed.

\section{The Credibility Analysis and Consistency Test of Prior Information}

2.1. The Reliability Evaluation Step of Low-Voltage Switchgear. The flowchart of reliability evaluation step of low-voltage switchgear was shown in Figure 1.

2.2. The Collection of Prior Information. The prior information of low-voltage switchgear is obtained mainly by two aspects.

(1) Historical Information. The information is obtained by historical data, and it is the most reliable and trustworthy way.

(2) Expert Opinion and Engineering Experience. Experts who work in the forefront production or design have a rich engineering experience, and they have a thorough understanding for low-voltage switchgear. The information can be obtained by the communication with them. It is unavoidable that there have some subjective components, but it accords with the project.

Manufacturers which dedicated low-voltage switchgear design development can be researched, and the entire user's feedback information can be collected. It is equivalent to gain the first-hand information from the production line. The historical data of low-voltage switchgear from the year 2007 to 2011 are shown in Figures 2-6.

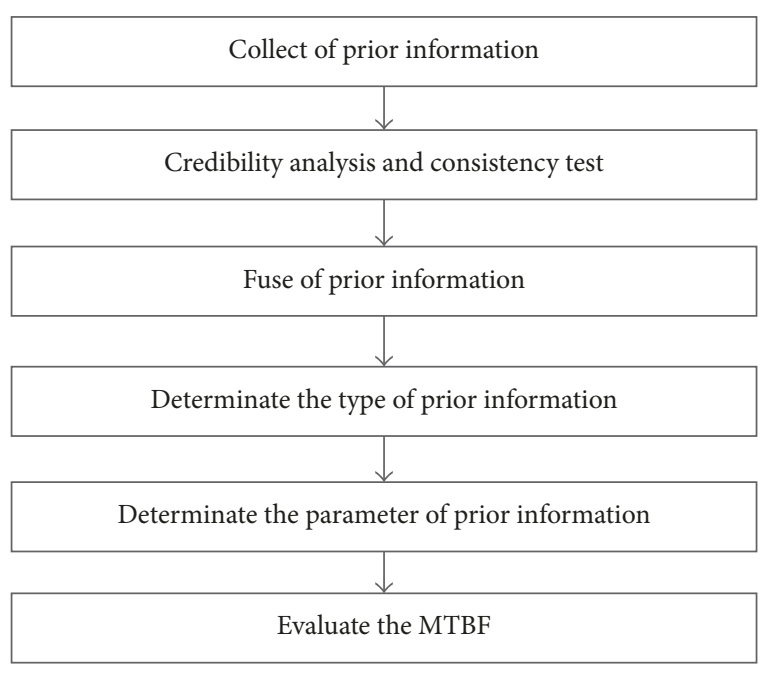

FIGURE 1: Reliability evaluation step of low-voltage switchgear.

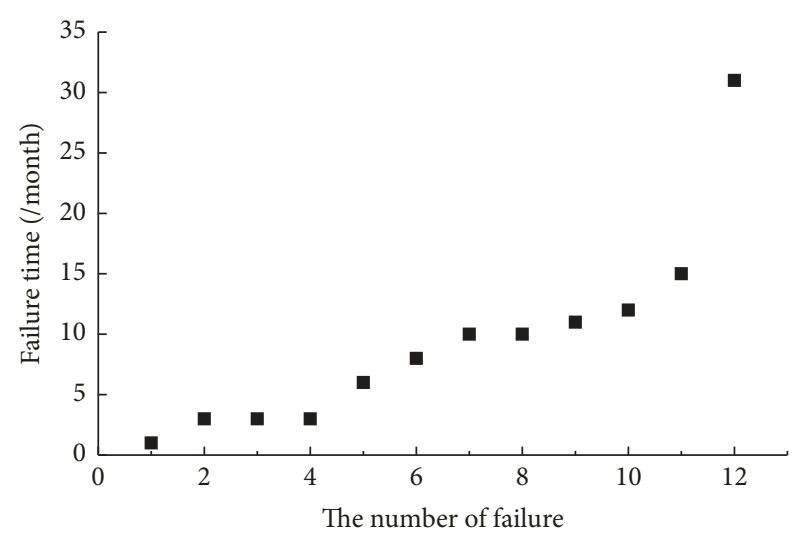

FIgURE 2: The historical data of the year 2007 of low-voltage switchgear.

Because of the difference of external environment and attainment methods, the prior information may not come from the same population distribution. And credibility analysis and consistency test are the key to this question [20]. Only credibility analysis and consistency test can ensure the validity of prior information.

2.3. The Credibility Analysis of Prior Information. Credibility analysis for prior information is important, because only credible prior information can reduce the risk in decision making.

It is denoted that $X=\left(x_{1}, x_{2}, \ldots, x_{m}\right)$ is the field test information and $F_{m}(x)$ is the distribution function, $Y=$ $\left(y_{1}, y_{2}, \ldots, y_{n}\right)$ is the prior information and $G_{n}(y)$ is the distribution function, and the two samples $X$ and $Y$ are independent.

To check whether the two types of information are the same population distribution, the hypotheses were as follows:

$H_{0}: X$ and $Y$ are the same population distribution.

$H_{1}: X$ and $Y$ are not the same population distribution. 


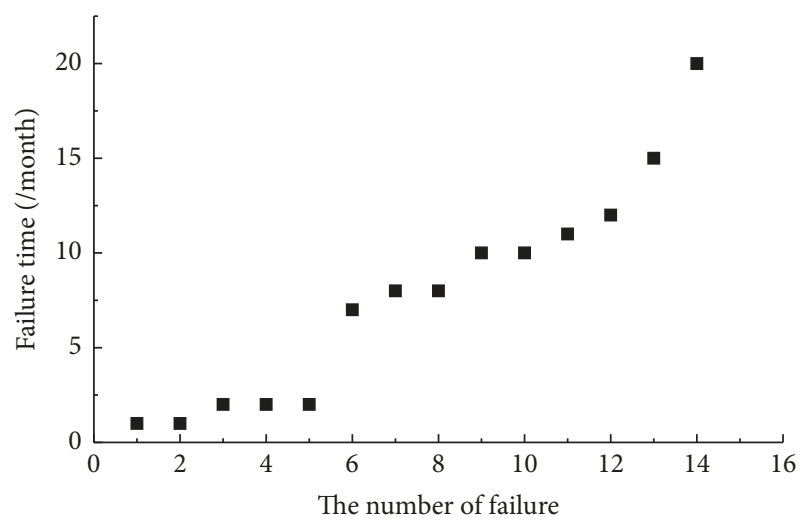

FIgURE 3: The historical data of the year 2008 of low-voltage switchgear.

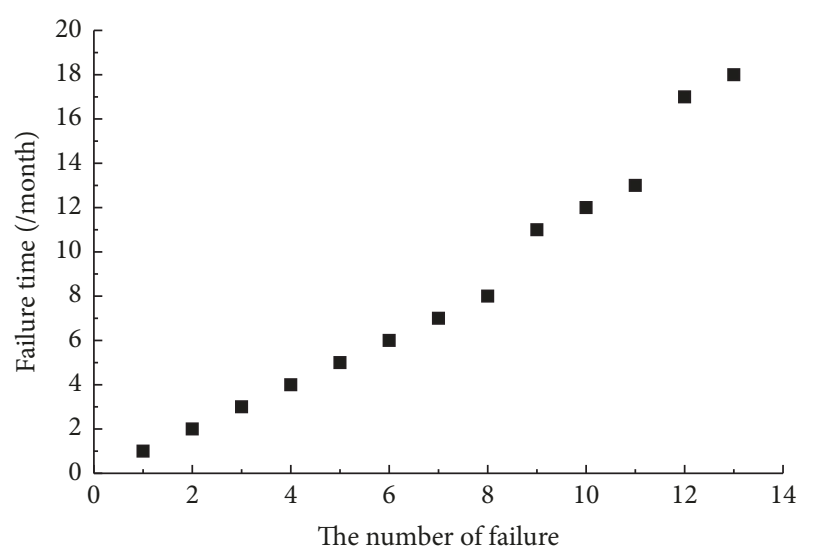

FIgURE 4: The historical data of the year 2009 of low-voltage switchgear.

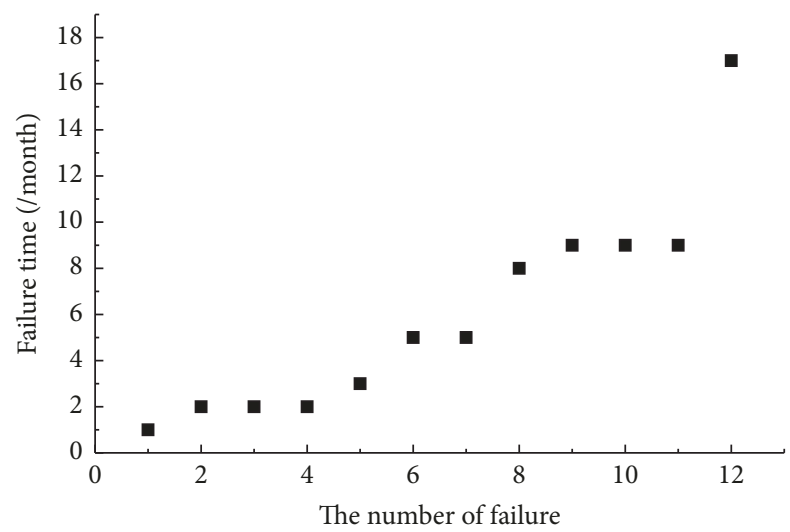

FIGURE 5: The historical data of the year 2010 of low-voltage switchgear.

To illustrate the concept of prior information credibility, it was denoted that

\section{$A$ accepts event $H_{0}$,}

$\bar{A}$ refuses event $H_{0}$ and accepts event $H_{1}$.

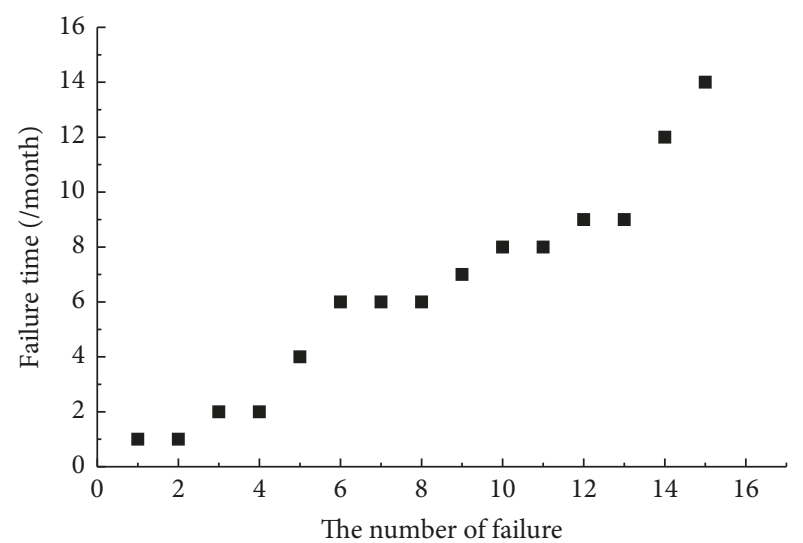

FIgURE 6: The historical data of the year 2011 of low-voltage switchgear.

TABLE 1: The prior probability $P\left(H_{0}\right)$ of the year from 2007 to 2011 .

\begin{tabular}{lccccc}
\hline Year & 2007 & 2008 & 2009 & 2010 & 2011 \\
\hline$P\left(H_{0}\right)$ & 0.55 & 0.55 & 0.6 & 0.7 & 0.82 \\
\hline
\end{tabular}

TABLE 2: The credibility of prior information.

\begin{tabular}{llllll}
\hline Year & 2007 & 2008 & 2009 & 2010 & 2011 \\
\hline$P\left(H_{0} \mid A\right)$ & 0.959 & 0.959 & 0.966 & 0.978 & 0.988 \\
\hline
\end{tabular}

Therefore, $P\left(A \mid H_{0}\right)=1-\alpha$ and $P\left(\bar{A} \mid H_{0}\right)=\alpha$, where $\alpha$ is the probability of rejection event $A$ when it is true.

Then the prior information credibility of $Y$ is

$$
\begin{aligned}
P & \left(H_{0} \mid A\right) \\
& =\frac{P\left(\bar{A} \mid H_{0}\right) P\left(H_{0}\right)}{P\left(A \mid H_{0}\right) P\left(H_{0}\right)+P\left(A \mid H_{1}\right)\left(1-P\left(H_{0}\right)\right)} \\
& =\left(1+\frac{\left(1-P\left(H_{0}\right)\right) \beta}{P\left(H_{0}\right)(1-\alpha)}\right)^{-1},
\end{aligned}
$$

where $\beta$ is the probability of the acception of event $A$ when it is false.

If the prior probability $P\left(H_{0}\right), \alpha$, and $\beta$ are known, the credibility of $Y$ can be obtained.

The prior probability $P\left(H_{0}\right)$ of every year was shown in Table 1. It is got according to the expert experience and historical data.

The risk equal principle [21,22] is used to confirm $\alpha$ and $\beta, \alpha=\beta=0.05$. Then the credibility of prior information from the year 2007 to 2011 was summarized in Table 2 .

From Table 2, it can be observed that the credibility of prior information of low-voltage switchgear is very high. And the higher credibility of prior information shows the higher confidence level of the reliability evaluation.

2.4. The Consistency Test of Prior Information. To use prior information accurately, the consistency between prior and field test information should be taken into account, if the 
TABLE 3: The results of Smirnov test.

\begin{tabular}{lcc}
\hline$X_{i}$ & $Y_{j}$ & $F_{m}(x)-G_{n}(y)$ \\
\hline 1 & 1 & $1 / 12-1 / 15=1 / 60$ \\
& 1 & $1 / 12-2 / 15=-3 / 60$ \\
2 & 2 & $2 / 12-2 / 15=2 / 60$ \\
2 & 2 & $3 / 12-3 / 15=-3 / 60$ \\
2 & & $4 / 12-3 / 15=8 / 60$ \\
3 & & $5 / 12-4 / 15=11 / 60$ \\
& 4 & $5 / 12-5 / 15=5 / 60$ \\
5 & & $6 / 12-5 / 15=10 / 60$ \\
5 & & $7 / 12-5 / 15=15 / 60$ \\
& 6 & $7 / 12-6 / 15=11 / 60$ \\
& 6 & $7 / 12-7 / 15=7 / 60$ \\
& 6 & $7 / 12-8 / 15=3 / 60$ \\
8 & 7 & $7 / 12-9 / 15=-1 / 60$ \\
& 8 & $8 / 12-10 / 15=0$ \\
9 & 8 & $8 / 12-11 / 15=-4 / 60$ \\
9 & 9 & $9 / 12-12 / 15=-3 / 60$ \\
9 & 9 & $10 / 12-13 / 15=-2 / 60$ \\
& & $11 / 12-13 / 15=3 / 60$ \\
& 12 & $11 / 12-14 / 15=-1 / 60$ \\
& 14 & $11 / 12-1=-5 / 60$ \\
& & $1-1=0$ \\
\hline
\end{tabular}

multiple historical data comes from different environment and stages. It is essential that prior information and field test information have the same population distribution approximately.

The Smirnov test method solves the problem of consistency between two samples. And the field test data was consistent with the prior information if the test statistic is out of the refused domain. The Smirnov test method was used to verify the consistency of low-voltage switchgear between the prior information and the field test data. $G_{n}(y)$.

The hypothesis is $H_{3}: F_{m}(x)=G_{n}(y)$, and $H_{4}: F_{m}(x) \neq$

The test statistics $D_{m+n}$ is

$$
D_{m+n}=\max \left|F_{m}(x)-G_{n}(y)\right| .
$$

The historical data of 2011 is the field test information, and the historical data of 2010 is the prior information. There are two hypotheses.

$\mathrm{H}_{5}$ : the field test information and the prior information are the same entire sample.

$\mathrm{H}_{6}$ : the field test information and the prior information are not the same entire sample.
The Smirnov test statistics of the two samples was shown in Table 3.

In Table 3, it can be known that $m=12, n=15$, $D_{m+n}=15 / 60=1 / 4$. Also, it can be observed that $P\left(D_{m+n}<\right.$ $23 / 60)=0.80$ from the attached list 10 in literature [23], and $P\left(D_{m+n}>1 / 4\right)>0.2$. Therefore, $H_{5}$ is accepted, and the field test information and the prior information are the same entire sample.

The historical data of 2011 is the field test information, and the historical data of 2007, 2008, 2009, and 2010 is the prior information, respectively. According to Smirnov test, the historical data of 2011 is the same entire sample with the year of 2007, 2008, 2009, and 2010.

\section{The Prior Distribution Type of Low-Voltage Switchgear}

The distribution type of prior information has a direct impact on reliability evaluation of low-voltage switchgear. In general, the reliability distributions of electrical products contain the following types, exponential distribution, normal distribution, and Weibull distribution $[24,25]$. Because of having three parameters, Weibull distribution can fit data precisely and contains exponential distribution and normal distribution. Therefore, it is assumed that the failure time of low-voltage switchgear obeys Weibull distribution.

The probability density distribution function of Weibull distribution [26] with three parameters is

$$
\begin{aligned}
& f(t ; \eta, m, \gamma)=\frac{m}{\eta}\left(\frac{t-\gamma}{\eta}\right)^{m-1} \exp \left(-\left(\frac{t-\gamma}{\eta}\right)^{m}\right) \\
& t>0
\end{aligned}
$$

where $\eta$ is the scale parameter, $m$ is the shape parameter, and $\gamma$ is the time extension function. In this paper, there is no time extension; that is to say, $\gamma$ is 0 .

Therefore, (3) is reduced to

$$
f(t ; \eta, m)=\frac{m}{\eta}\left(\frac{t}{\eta}\right)^{m-1} \exp \left(-\left(\frac{t}{\eta}\right)^{m}\right), \quad t>0 .
$$

The historical data was ordered from small to large $t_{1} \leq$ $t_{2} \leq \cdots \leq t_{i} \leq \cdots \leq t_{r}$, and $X_{i}=\ln t_{i}$. Then the test statistics $W$ is

$$
W=\frac{\sum_{i=r_{1}+1}^{r-1}\left(k_{i} /\left(r-r_{1}-1\right)\right)}{\sum_{i=1}^{r_{i}}\left(t_{i} / r_{i}\right)}
$$

where

$$
\begin{aligned}
& r_{1}= \begin{cases}\frac{r}{2}, & \text { when } r \text { is a even number } \\
\frac{(r-1)}{2}, & \text { when } r \text { is an add number }\end{cases} \\
& k_{i}=\frac{\left[x_{i+1}-x_{i}\right]}{\ln [\ln ((4(r-i-1)+3) /(4 r+1)) / \ln ((4(r-i)+3) /(4 r+1))]} .
\end{aligned}
$$


When the significance level is $\alpha$, if the prior information of low-voltage switchgear obeys Weibull distribution, the $W$ should obey

$$
\begin{aligned}
& F_{\alpha / 2}\left(2\left(r-r_{1}-1\right), 2 r_{1}\right) \leq W \\
& \quad \leq F_{1-\alpha / 2}\left(2\left(r-r_{1}-1\right), 2 r_{1}\right) .
\end{aligned}
$$

According to data in Figure 6, it can be seen that $r=12$, $r_{1}=6$. If $\alpha=0.05$, it can be calculated that $W=$ $0.3502, F_{0.975}(10,12)=2.91, F_{0.025}(10,12)=0.3436$, and $F_{0.025}(10,12)<W<F_{0.975}(10,12)$. Therefore, the prior information in 2011 obeys Weibull distribution.

\section{The Maximum Entropy Method for the Prior Distribution}

4.1. Prior Distribution of Low-Voltage Switchgear. Formula (8) is the expression of the joint prior distribution $\pi(\eta, m)$, and formula (9) to formula (11) are the constraint conditions.

$$
\begin{aligned}
& \max H(\eta, m) \\
& =-\int_{-\infty}^{+\infty} \int_{-\infty}^{+\infty} \pi(\eta, m) \ln \pi(\eta, m) d \eta d m \\
& \int_{-\infty}^{+\infty} \int_{-\infty}^{+\infty} \pi(\eta, m) d \eta d m=1 \\
& \int_{-\infty}^{+\infty} \int_{-\infty}^{+\infty} \eta^{i} \pi(\eta, m) d \eta d m=E\left(\eta^{i}\right)=\eta_{i}, \\
& \int_{-\infty}^{+\infty} \int_{-\infty}^{+\infty} m^{j} \pi(\eta, m) d \eta d m=E\left(m^{j}\right)=m_{j}, \\
& \quad j=1, \ldots, k \\
& \quad j=1, \ldots, n,
\end{aligned}
$$

where $\eta_{i}, m_{j}$ are the origin moments of $i$ order and $j$ order of two-parameter $\eta$ and $m$, respectively; $k$ and $n$ are the highest order of two-parameter origin moment, respectively.

To solve $\pi(\eta, m)$, the following auxiliary function was constructed:

$$
\begin{array}{r}
J[\pi(\eta, m)]=\int_{-\infty}^{+\infty} \int_{-\infty}^{+\infty}(-\pi(\eta, m) \ln \pi(\eta, m) \\
\left.+\sum_{i=0}^{k} \lambda_{i} \eta^{i} \pi(\eta, m)+\sum_{i=0}^{k} c_{j} \eta^{i} \pi(\eta, m)\right) d \eta d m,
\end{array}
$$

where $\lambda_{i}$ and $c_{j}$ were the Lagrange Multiplier.

When $\partial J[\pi(\eta, m)] / \partial \pi(\eta, m)=0$, it can be solved that

$$
\pi(\eta, m)=\exp \left(\lambda_{0}-1+\sum_{i=1}^{k} \lambda_{i} \eta^{i}+\sum_{j=1}^{n} c_{j} m^{j}\right) .
$$

According to the expert experience, $k=n=2$,

$$
\begin{gathered}
\pi(\eta, m)=\exp \left(\lambda_{0}-1-\frac{\lambda_{1}^{2}}{4 \lambda_{2}}-\frac{c_{1}^{2}}{4 c_{2}}\right. \\
\left.+\lambda_{2}\left(\eta+\frac{\lambda_{1}}{2 \lambda_{2}}\right)^{2}+c_{2}\left(m+\frac{c_{1}}{2 c_{2}}\right)^{2}\right) .
\end{gathered}
$$

On the constraints, by joint density function normalization, $\pi(\eta, m)$ obeys bivariate normal distribution as

$$
\pi(\eta, m)=\frac{1}{2 \pi \sigma_{\eta} \sigma_{m}} \exp \left(-\frac{(\eta-\bar{\eta})^{2}}{2 \sigma_{\eta}^{2}}-\frac{(m-\bar{m})^{2}}{2 \sigma_{m}^{2}}\right),
$$

where $\bar{\eta}, \bar{m}, \sigma_{\eta}^{2}$, and $\sigma_{m}^{2}$ were the mathematical expectation and the variance of $\eta$ and $m$.

From (15), it can be known that the prior distribution not only contains the known prior information but tries to avoid the introduction of other assumption information.

4.2. The Determination of Parameters. In (15), it contains two parameters $\eta$ and $m$. And to obtain $\bar{\eta}, \bar{m}, \sigma_{\eta}^{2}$, and $\sigma_{m}^{2}$, the bootstrap method [27-30] was used.

$T=\left(t_{1}, t_{2}, \ldots, t_{n}\right)$ denotes a set of obtained samples with total failure. The distribution parameter samples can be obtained by the following steps.

(1) The self-help sample $T^{*}=\left(t_{1}^{*}, t_{2}^{*}, \ldots, t_{n}^{*}\right)$ can be obtained by carrying out sampling with replacement for $T=$ $\left(t_{1}, t_{2}, \ldots, t_{n}\right)$.

(2) $\widehat{m}$ and $\widehat{\eta}$ can be got from the maximum likelihood estimation with the self-help sample $T^{*}=\left(t_{1}^{*}, t_{2}^{*}, \ldots, t_{n}^{*}\right)$.

(3) Repeat the previous two steps for $N$ times; then the estimated parametric sample can be got.

$$
\left\{\left(\widehat{m}_{1}, \widehat{\eta}_{1}\right),\left(\widehat{m}_{2}, \widehat{\eta}_{2}\right), \ldots,\left(\widehat{m}_{N}, \widehat{\eta}_{N}\right)\right\}
$$

(4) The expectation and variance of the unknown parameter $m$ and $\eta$ can be got by the estimated parametric samples.

$$
\begin{aligned}
\bar{\eta} & =\frac{1}{n} \sum_{i=1}^{n} \widehat{\eta}_{i}, \\
\sigma_{\eta}^{2} & =\frac{1}{n-1} \sum_{i=1}^{n}\left(\widehat{\eta}_{i}-\bar{\eta}\right)^{2} \\
\bar{m} & =\frac{1}{n} \sum_{j=1}^{n} \widehat{m}_{j}, \\
\sigma_{m}^{2} & =\frac{1}{n-1} \sum_{j=1}^{n}\left(\widehat{m}_{j}-\bar{m}\right)^{2} .
\end{aligned}
$$

By calculating the transcendental moment, the parameter expressions of the prior distribution can be got,

$$
\begin{aligned}
\bar{m} & =\overline{\widehat{m}}, \\
\sigma_{m}^{2} & =S_{m}^{2},
\end{aligned}
$$




$$
\begin{aligned}
\bar{\eta} & =\overline{\hat{\eta}}, \\
\sigma_{\eta}^{2} & =S_{\eta}^{2} .
\end{aligned}
$$

Input the data in Figures 2-6 to the MATLAB; the selfhelp sample can be got by the bootstrap method. And the maximum likelihood estimate can be got by the wblfit() command in MATLAB. Finally, the mathematical expectation and the variance of the two parameters are

$$
\begin{aligned}
\bar{\eta} & =6145.5, \\
\bar{m} & =1.4000 \\
\sigma_{\eta}^{2} & =9832.0, \\
\sigma_{m}^{2} & =0.0016
\end{aligned}
$$

The prior density function of low-voltage switchgear is

$$
\begin{aligned}
& \pi(\eta, m)=\frac{1}{7.9325 \pi} \\
& \cdot \exp \left(-\frac{(\eta-6145.5)^{2}}{19.664}-\frac{(m-1.4000)^{2}}{0.0032}\right)
\end{aligned}
$$

\section{The MTBF Evaluation of Low-Voltage Switchgear}

Based on Bayesian principle, the field life data of low-voltage switchgear can be assumed as $T=\left(t_{1}, t_{2}, \ldots, t_{n}\right)$, and then its likelihood function can be expressed as

$$
\begin{aligned}
& p\left(t_{1}, t_{2}, \ldots, t_{n} \mid \eta, m\right) \\
& \quad=\left(\frac{m}{\eta}\right)^{n} \prod_{i=1}^{n}\left(\frac{t_{i}}{\eta}\right)^{m-1} \exp \left(-\sum_{i=1}^{n}\left(\frac{t_{i}}{\eta}\right)^{m}\right) .
\end{aligned}
$$

Then the joint posterior distribution of parameter $\eta, m$ can be expressed as

$$
\begin{aligned}
& h\left(\eta, m \mid t_{1}, t_{2}, \ldots, t_{n}\right) \\
& \quad=\frac{p\left(t_{1}, t_{2}, \ldots, t_{n} \mid \eta, m\right) \pi(\eta, m)}{\int_{-\infty}^{+\infty} \int_{-\infty}^{+\infty} p\left(t_{1}, t_{2}, \ldots, t_{n} \mid \eta, m\right) \pi(\eta, m) d \eta d m} .
\end{aligned}
$$

By the numerical calculation, the point estimate of the posterior distribution can be obtained,

$$
\begin{gathered}
\widehat{m}=E\left(m \mid t_{1}, t_{2}, \ldots, t_{n}\right)=1.1640 \\
\widehat{\eta}=E\left(\eta \mid t_{1}, t_{2}, \ldots, t_{n}\right)=7021.3 .
\end{gathered}
$$

Finally, the estimation value of MTBF of low-voltage switchgear is

$$
\overline{\mathrm{MTBF}}=\widehat{\eta} \Gamma\left(1+\frac{1}{\widehat{m}}\right)=6659.06 \mathrm{~h}
$$

\section{Conclusion}

A maximum entropy multisource information fusion method was proposed to obtain the prior information of low-voltage switchgear and then evaluate the reliability. The proposed method fused the expert experience, engineering experience, and historical data. And the method was illustrated by historical data of the year from 2007 to 2011 and experience of low-voltage switchgear.

The creditability analysis and the compatibility test of the prior information were presented by the Smirnov test method. The higher credibility of prior information shows the higher confidence level of historical data and experience. Also, it is a solid basic for the reliability evaluation of lowvoltage switchgear

The type of the prior information was determined and the parameter was solved by maximum entropy. It obeys bivariate normal prior distribution. The estimation values of the two parameters are 6145.5 and 1.4000. According to Bayesian theory, the point estimations of the posterior distribution of the two parameters are 7021.3 and 1.1640. Finally, the estimation value of posterior MTBF of lowvoltage switchgear is $6659.03 \mathrm{~h}$, which is about 0.76 years. It is a waste to humans in effort, time, and finance. The evaluation result reduces the experimental period and test cost, which is an improvement for the reliability evaluation and management of low-voltage switchgear and also an improvement for other systems with simple sample data.

\section{Conflicts of Interest}

The authors declare that they have no conflicts of interest.

\section{Acknowledgments}

This work was supported by the National Natural Science Foundation of China (no. 51077039) and Hebei Province Natural Science Foundation of China (no. E2016202134).

\section{References}

[1] S. Y. Xu, L. Zhang, and J. Q. Wang, "Study on prior information fusion method in reliability assessment for low-voltage switchgear," in Proceedings of the in Proceeding of the 26th International Conference on Electrical Contacts, pp. 452-455, China, May 2012.

[2] C. Zhou, Study of condition monitoring and comprehensive diagnosis for middle-voltage and low-voltage switchgear, Chongqing University, 2016.

[3] B. Cai, H. Liu, and M. Xie, "A real-time fault diagnosis methodology of complex systems using object-oriented Bayesian networks," Mechanical Systems and Signal Processing, vol. 80, pp. 31-44, 2016.

[4] B. Cai, Y. Liu, Q. Fan et al., "Multi-source information fusion based fault diagnosis of ground-source heat pump using Bayesian network," Applied Energy, vol. 114, pp. 1-9, 2014.

[5] B. P. Cai, M. Xie, and Y. H. Liu, "Availability-based engineering resilience and its corresponding evaluation methodology," Reliability engineering system safety, vol. 172, pp. 216-224, 2018. 
[6] B. Cai, L. Huang, and M. Xie, "Bayesian Networks in Fault Diagnosis," IEEE Transactions on Industrial Informatics, vol. 13, no. 5, pp. 2227-2240, 2017.

[7] H.-Z. Huang, P.-L. Cui, W. Peng, H.-Y. Gao, and H.-K. Wang, "Fatigue lifetime assessment of aircraft engine disc via multisource information fusion," International Journal of Turbo and Jet Engines, vol. 31, no. 2, pp. 167-174, 2014.

[8] I. Rigas, E. Abdulin, and O. Komogortsev, "Towards a multisource fusion approach for eye movement-driven recognition," Information Fusion, vol. 32, pp. 13-25, 2016.

[9] F. Wang, L. Hu, and J. Zhou, "A semantics-based approach to multi-source heterogeneous information fusion in the internet of things," Soft Computing, vol. 21, pp. 2005-2013, 2017.

[10] G. Q. Zhao, W. Liang, and S. Y. He, "Empirical Entropy for right censored data," Acta mathematicae applicatae sinica-english series, vol. 31, pp. 395-404, 2015.

[11] A. K. Singh, H. P. Singh, and Karmeshu, "Analysis of finite buffer queue: Maximum entropy probability distribution with shifted fractional geometric and arithmetic means," IEEE Communications Letters, vol. 19, no. 2, pp. 163-166, 2015.

[12] K. N. Oikonomou and P. D. Grünwald, "Explicit bounds for entropy concentration under linear constraints," IEEE Transactions on Information Theory, vol. 62, no. 3, pp. 1206-1230, 2016.

[13] B. Grechuk, A. Molyboha, and M. Zabarankin, "Maximum entropy principle with general deviation measures," Mathematics of Operations Research, vol. 34, no. 2, pp. 445-467, 2009.

[14] R. R. Yager and F. Petry, "An intelligent quality-based approach to fusing multi-source probabilistic information," Information Fusion, vol. 31, pp. 127-136, 2016.

[15] R. I. Al-Ruzouq, "Data fusion of multi-source imagery based on linear features registration," International Journal of Remote Sensing, vol. 31, no. 19, pp. 5011-5021, 2010.

[16] G. N. Boshnakov and S. Lambert-Lacroix, "A periodic Levinson-Durbin algorithm for entropy maximization," Computational Statistics \& Data Analysis, vol. 56, no. 1, pp. 15-24, 2012.

[17] A. Dukkipati, S. Bhatnagar, and M. N. Murty, "On measuretheoretic aspects of nonextensive entropy functionals and corresponding maximum entropy prescriptions," Physica A: Statistical Mechanics and its Applications, vol. 384, no. 2, pp. 758-774, 2007.

[18] A. Caticha and R. Preuss, "Maximum entropy and Bayesian data analysis: Entropic prior distributions," Spatial Statistics, vol. 23, pp. 91-108, 2004.

[19] H.-J. Kim, "A two-stage maximum entropy prior of location parameter with a stochastic multivariate interval constraint and its properties," Entropy, vol. 18, no. 5, article no. 188, 2016.

[20] J. Ye, "Interval Neutrosophic Multiple Attribute DecisionMaking Method with Credibility Information," International Journal of Fuzzy Systems, vol. 18, no. 5, pp. 914-923, 2016.

[21] H. G. Claycamp, "Rapid benefit-risk assessments: No escape from expert judgments in risk management," Risk Analysis, vol. 26, no. 1, pp. 147-156, 2006.

[22] S. Weber, "Distribution-invariant risk measures, entropy, and large deviations," Journal of Applied Probability, vol. 44, no. 1, pp. 16-40, 2007.

[23] S. X. Zhong, J. H. Yan, Y. P. Cheng et al., The commonly used statistical tables, China statistics press, Beijing, China, 1990.

[24] J. G. Lu, Electrical reliability theory and application, China machine press, Beijing, China, 1996.
[25] G. W. Li, Reliability engineering outline, National defense industry press, Beijing, China, 1989.

[26] L. Li, D. Ma, and Z. Li, "Cox-proportional hazards modeling in reliability analysis - A study of electromagnetic relays data," IEEE Transactions on Components, Packaging, and Manufacturing Technology, vol. 5, no. 11, pp. 1582-1589, 2015.

[27] G. Cheng, "Moment Consistency of the Exchangeably Weighted Bootstrap for Semiparametric M-estimation," Scandinavian Journal of Statistics, vol. 42, no. 3, pp. 665-684, 2015.

[28] J.-P. Kreiss and E. Paparoditis, "Bootstrap methods for dependent data: a review," Journal of the Korean Statistical Society, vol. 40, no. 4, pp. 357-378, 2011.

[29] C.-H. Wang, "An intuitionistic fuzzy set-based hybrid approach to the innovative design evaluation mode for green products," Advances in Mechanical Engineering, vol. 8, no. 4, pp. 1-16, 2016.

[30] C.-H. Wang, M.-L. Tseng, K.-H. Tan, and K.-T. Yu, "Application of a mathematical programming model to solve the confidence interval of process capability index Spk," International Journal of Information and Management Sciences, vol. 28, no. 1, pp. 11-23, 2017. 


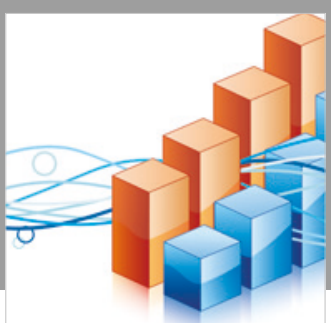

Advances in

Operations Research

\section{-n-m}
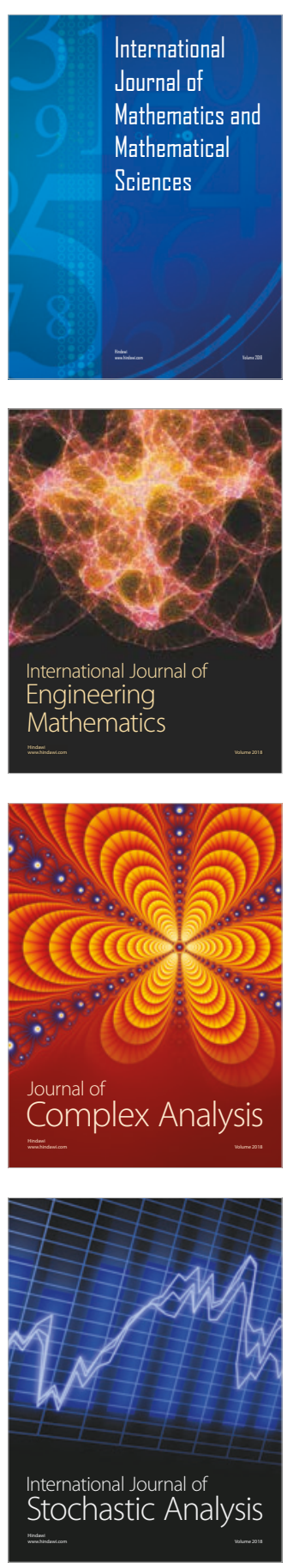
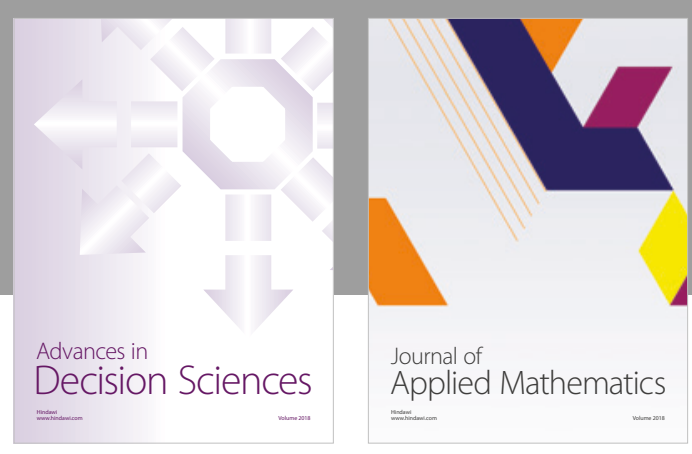

Journal of

Applied Mathematics
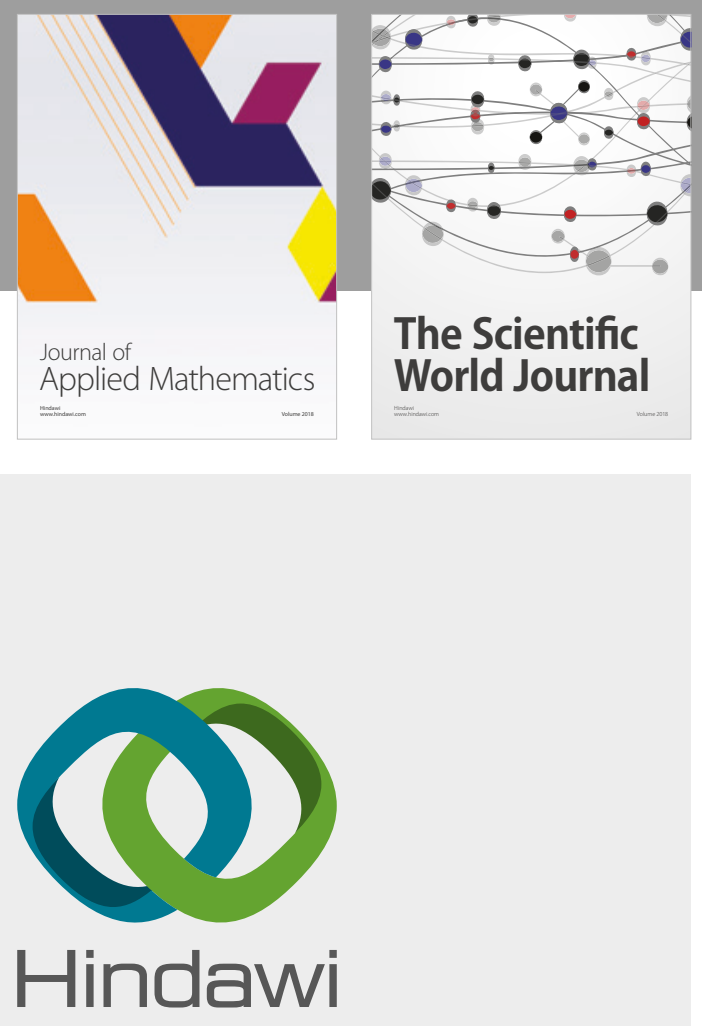

Submit your manuscripts at

www.hindawi.com

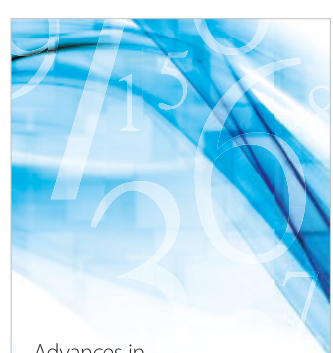

Advances in
Numerical Analysis
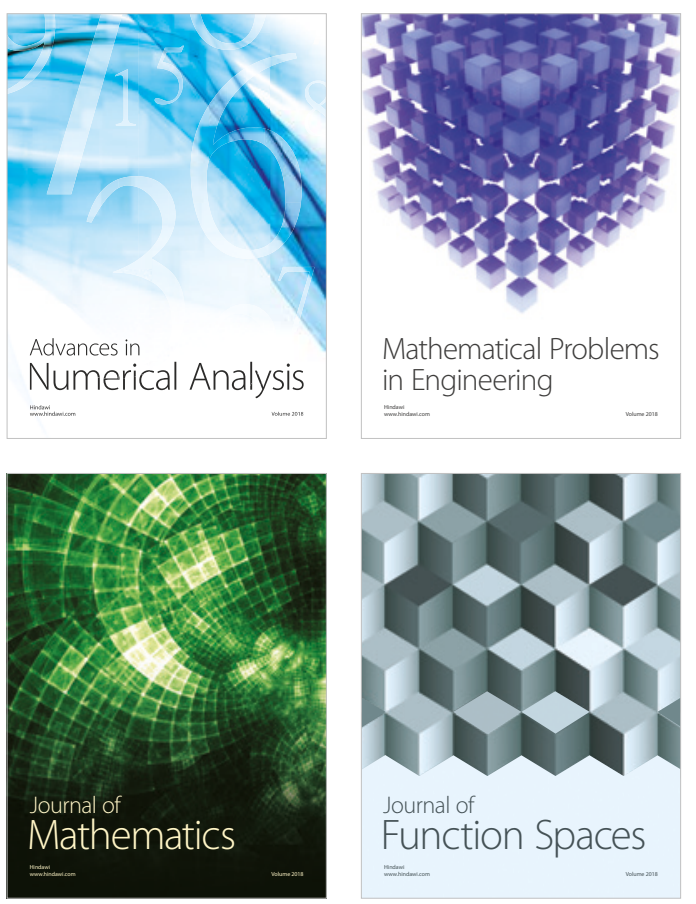

Mathematical Problems in Engineering

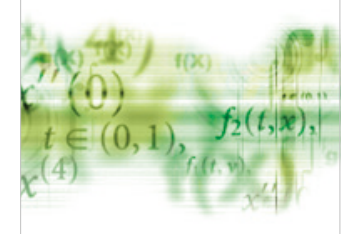

International Journal of

Differential Equations

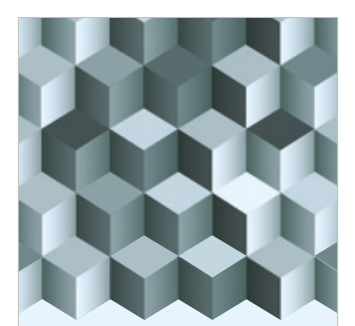

Journal of

Function Spaces

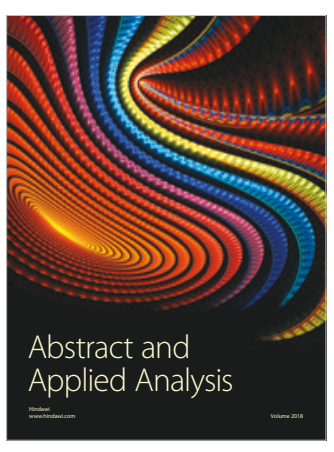

The Scientific

World Journal

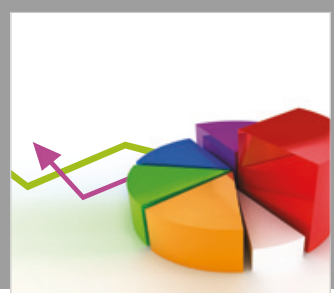

Journal of

Probability and Statistics
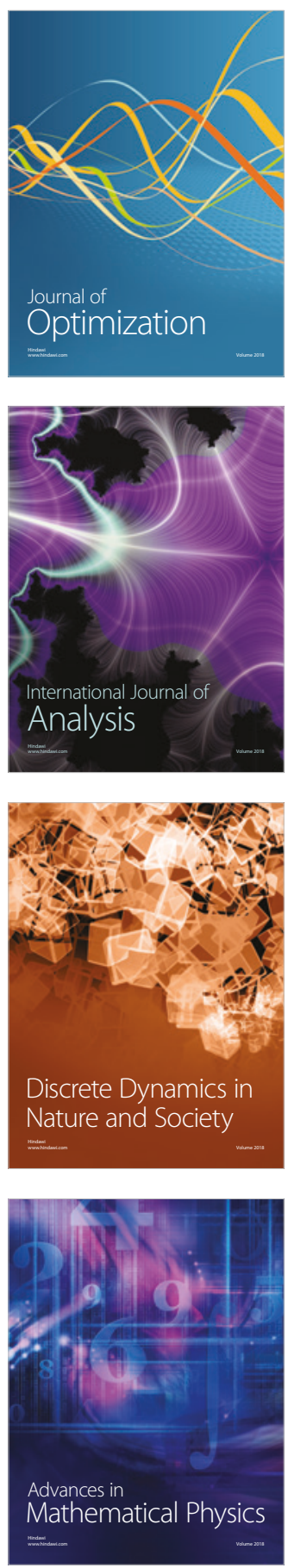